PROCEEDINGS OF THE

AMERICAN MATHEMATICAL SOCIETY

Volume 137, Number 1, January 2009, Pages 329-337

S 0002-9939(08)09509-9

Article electronically published on May 15, 2008

\title{
EXPONENTIAL ERGODICITY OF NON-LIPSCHITZ STOCHASTIC DIFFERENTIAL EQUATIONS
}

\author{
XICHENG ZHANG
}

(Communicated by Richard C. Bradley)

\begin{abstract}
Using the coupling method and Girsanov's theorem, we study the strong Feller property and irreducibility for the transition probabilities of stochastic differential equations with non-Lipschitz and monotone coefficients. Then, the exponential ergodicity and the spectral gap for the corresponding transition semigroups are obtained under fewer assumptions.
\end{abstract}

\section{INTRODUCTION AND MAIN RESULT}

Consider the following stochastic differential equation (SDE):

$$
\mathrm{d} X_{t}=b\left(X_{t}\right) \mathrm{d} t+\sigma\left(X_{t}\right) \mathrm{d} W_{t}, \quad X_{0}=x_{0} \in \mathbb{R}^{d},
$$

where $b: \mathbb{R}^{d} \rightarrow \mathbb{R}^{d}$ and $\sigma: \mathbb{R}^{d} \rightarrow \mathbb{R}^{d} \times \mathbb{R}^{d}$ are continuous functions and $\left(W_{t}\right)_{t \geqslant 0}$ is a $d$-dimensional standard Brownian motion defined on some complete probability space $(\Omega, \mathcal{F}, \mathbf{P})$.

When $b$ and $\sigma$ are locally Lipschitz continuous and monotonic, the asymptotic behavior of diffusion process $X_{t}$ as $t \rightarrow \infty$ is well studied (see for example [2]). Recently, since the work of Malliavin in [8] and LeJan-Raimond in 7], there are increasing interests for the studies of non-Lipschitz SDE, in particular, when the coefficients have $r\left(1 \vee \log r^{-1}\right)^{1 / 2}$-type continuous modulus. For this type of SDE, the flow property and large deviation estimate have been obtained in 1, 15, 15, 10, 11. In this work, we shall concentrate on the study of the exponential ergodicity of solutions to this type of non-Lipschitz SDE.

Let us first recall some notions about the ergodicity (for example, see 2, Chapter 2]). Let $\left\{X_{t}\left(x_{0}\right), t \geqslant 0, x_{0} \in \mathbb{E}\right\}$ be a family of Markov processes on some probability space $(\Omega, \mathcal{F}, \mathbf{P})$, where $\mathbb{E}$ stands for the state space and is usually a Polish space. That defines a family of transition probabilities on Borel measurable space $(\mathbb{E}, \mathscr{B}(\mathbb{E}))$ :

$$
P_{t}\left(x_{0}, E\right):=\mathbf{P}\left(X_{t}\left(x_{0}\right) \in E\right), \quad E \in \mathscr{B}(\mathbb{E}) .
$$

Received by the editors August 6, 2007, and, in revised form, December 15, 2007.

2000 Mathematics Subject Classification. Primary 60H10, 37A25.

Key words and phrases. Strong Feller property, irreducibility, ergodicity, spectral gap, nonLipschitz stochastic differential equation.

(C)2008 American Mathematical Society Reverts to public domain 28 years from publication 
An invariant measure $\mu$ is defined as a stationary distribution of $P_{t}$, i.e.:

$$
\int_{\mathbb{E}} P_{t}\left(x_{0}, E\right) \mu\left(\mathrm{d} x_{0}\right)=\mu(E), \quad \forall t>0, E \in \mathscr{B}(\mathbb{E}) .
$$

The existence of such an invariant measure has been considered extensively for various systems (cf. 2, 3], etc.). But, the uniqueness (or ergodicity) of invariant measures is a more difficult problem. A standard method called the "overlap method" for the uniqueness of invariant measures of $P_{t}$ is the following Doob-Khasminskii theorem (cf. 2]):

If $P_{t}$ is strong Feller and irreducible, then $P_{t}$ admits at most one invariant measure.

Recall that $P_{t}$ is strong Feller if for each $t>0$ and $E \in \mathscr{B}(\mathbb{E})$

$$
\mathbb{E} \ni x_{0} \mapsto P_{t}\left(x_{0}, E\right) \in[0,1] \text { is continuous; }
$$

$P_{t}$ is irreducible if for each $t>0$ and $x_{0} \in \mathbb{E}$

$$
P_{t}\left(x_{0}, E\right)>0 \text { for any non-empty open set } E \subset \mathbb{E} \text {. }
$$

For a dynamical system determined by an SDE with smooth coefficients, the strong Feller property is a direct consequence of the hypoellipticity of a diffusion operator associated with the SDE, which asserts by Hörmander's theorem that the transition probability has a smooth density with respect to the Lebesgue measure. Another efficient tool for proving the strong Feller property is the Bismut formula (cf. 2]). However, these two arguments seem to be hardly used to deal with the non-Lipschitz SDEs.

In order to prove the strong Feller property for non-Lipschitz SDEs, we shall use the coupling method combined with the Girsanov transformation. This method has been used in 13 to prove a Harnack type inequality for stochastic porous medium equations. An obvious advantage of this method lies in the succinctness of the proof. Here, the key point for us is the suitable choice of a coupling function. Moreover, for the irreducibility we shall use the ideas of the approximative controllability and Girsanov's transformation. The novelty of this paper is the use of Girsanov's theorem in the proof of the strong Feller property and irreducibility.

Before stating our main result, we introduce the following notations: Let $\langle\cdot, \cdot\rangle$ denote the inner product in $\mathbb{R}^{d},|\cdot|$ the length of a vector in $\mathbb{R}^{d}$, and $\|\cdot\|_{2}$ the Hilbert-Schmit norm from $\mathbb{R}^{d}$ to $\mathbb{R}^{d}$. Let $B_{b}\left(\mathbb{R}^{d}\right)$ be the Banach space of all bounded measurable functions on $\mathbb{R}^{d}$; the norm in $B_{b}\left(\mathbb{R}^{d}\right)$ is denoted by $\|\cdot\|_{0}$.

We make the following assumptions on the continuous coefficients $b$ and $\sigma$ :

(H1) (Monotonicity) There exists a $\lambda_{0} \in \mathbb{R}$ such that for all $x, y \in \mathbb{R}^{d}$

$$
\begin{aligned}
& 2\langle x-y, b(x)-b(y)\rangle+\|\sigma(x)-\sigma(y)\|_{2}^{2} \\
\leqslant & \lambda_{0}|x-y|^{2}\left(1 \vee \log |x-y|^{-1}\right) .
\end{aligned}
$$

(H2) (Growth of $\sigma$ ) There exists a $\lambda_{1}>0$ such that for all $x \in \mathbb{R}^{d}$

$$
\|\sigma(x)\|_{2} \leqslant \lambda_{1}(1+|x|) .
$$

(H3) (Non-degeneracy of $\sigma$ ) For some $\lambda_{2}>0$

$$
\sup _{x \in \mathbb{R}^{d}}\left\|\sigma^{-1}(x)\right\|_{2} \leqslant \lambda_{2} \text {. }
$$


(H4) (One side growth of $b$ ) There exist a $p>2$ and constants $\lambda_{3}, \lambda_{4} \geqslant 0$ such that for all $x \in \mathbb{R}^{d}$

$$
2\langle x, b(x)\rangle+\|\sigma(x)\|_{2}^{2} \leqslant-\lambda_{3}|x|^{p}+\lambda_{4} .
$$

It is well known that under (H1) and (H2), equation (11) has a unique continuous strong solution (cf. [12), which is denoted by $X_{t}\left(x_{0}\right)$. The transition semigroup associated with $X_{t}\left(x_{0}\right)$ is defined by

$$
P_{t} \varphi\left(x_{0}\right):=\mathbf{E} \varphi\left(X_{t}\left(x_{0}\right)\right), \quad t>0, \quad \varphi \in B_{b}\left(\mathbb{R}^{d}\right) .
$$

The transition probability is given by

$$
P_{t}\left(x_{0}, E\right):=\left(P_{t} 1_{E}\right)\left(x_{0}\right)=\mathbf{P}\left(X_{t}\left(x_{0}\right) \in E\right), \quad E \in \mathscr{B}\left(\mathbb{R}^{d}\right) .
$$

We are now in a position to state our main result in the present paper.

Theorem 1.1. Assume (H1)-(H3). Then the semigroup $P_{t}$ is strong Feller and irreducibility. If in addition, $(\mathbf{H} 4)$ holds, then there exists a unique invariant probability measure $\mu$ of $P_{t}$ having full support in $\mathbb{R}^{d}$ such that

(i) If $\lambda_{3}=0$ in $(\mathbf{H} 4)$, then for all $t>0$ and $x_{0} \in \mathbb{R}^{d}, \mu$ is equivalent to $P_{t}\left(x_{0}, \cdot\right)$, and

$$
\lim _{t \rightarrow \infty}\left\|P_{t}\left(x_{0}, \cdot\right)-\mu\right\|_{\text {Var }}=0
$$

where $\|\cdot\|_{\text {Var }}$ denotes the total variation of a signed measure.

(ii) If $\lambda_{3}>0$ in $(\mathbf{H} 4)$, then for some $\alpha, C>0$ independent of $x_{0}$ and $t$,

$$
\left\|P_{t}\left(x_{0}, \cdot\right)-\mu\right\|_{\mathrm{Var}} \leqslant C \cdot e^{-\alpha t} .
$$

Moreover, for any $q>1$ and each $\varphi \in L^{q}\left(\mathbb{R}^{d}, \mu\right)$

$$
\left\|P_{t} \varphi-\mu(\varphi)\right\|_{L^{q}\left(\mathbb{R}^{d}, \mu\right)} \leqslant C_{q} \cdot e^{-\alpha t / q}\|\varphi\|_{L^{q}\left(\mathbb{R}^{d}, \mu\right)}, \quad \forall t>0,
$$

where $\alpha$ is the same as above and $\mu(\varphi):=\int_{\mathbb{R}^{d}} \varphi(x) \mu(\mathrm{d} x)$. In particular, let $L_{q}$ be the generator of $P_{t}$ in $L^{q}\left(\mathbb{R}^{d}, \mu\right)$; then $L_{q}$ has a spectral gap (greater than $\alpha / q)$ in $L^{q}\left(\mathbb{R}^{d}, \mu\right)$.

This theorem will be proved in the next section.

\section{Proof of the main Result}

We need the following generalization of the Gronwall-Belmman type inequality (cf. [15]).

Lemma 2.1 (Bihari's inequality). Let $\rho_{\eta}: \mathbb{R}^{+} \mapsto \mathbb{R}^{+}$be a concave function given by

$$
\rho_{\eta}(x):= \begin{cases}x \log x^{-1}, & x \leqslant \eta, \\ \eta \log \eta^{-1}+\left(\log \eta^{-1}-1\right)(x-\eta), & x>\eta,\end{cases}
$$

where $\eta>0$. If $g(s), q(s)$ are two strictly positive functions on $\mathbb{R}^{+}$such that

$$
g(t) \leqslant g(0)+\int_{0}^{t} q(s) \rho_{\eta}(g(s)) d s, t \geqslant 0,
$$

then

$$
g(t) \leqslant(g(0))^{\exp \left\{-\int_{0}^{t} q(s) d s\right\}} .
$$

We shall separately prove the strong Feller property and irreducibility in the next two subsections, and assume that (H1)-(H3) hold. 
2.1. Strong Feller properties. In the following, for the sake of simplicity, for $z \neq 0 \in \mathbb{R}^{d}$ we write

$$
\bar{z}:=z /|z| \text {. }
$$

Let us now consider the following coupling SDEs:

$$
\begin{cases}\mathrm{d} X_{t}=b\left(X_{t}\right) \mathrm{d} t+\sigma\left(X_{t}\right) \mathrm{d} W(t), & X_{0}=x_{0}, \\ \mathrm{~d} Y_{t}=b\left(Y_{t}\right) \mathrm{d} t+a\left(X_{t}-Y_{t}\right) \cdot 1_{\{t<\tau\}} \mathrm{d} t+\sigma\left(Y_{t}\right) \mathrm{d} W_{t}, & Y_{0}=y_{0},\end{cases}
$$

where $a$ is the coupling function defined by

$$
a(z):=\left|x_{0}-y_{0}\right|^{\alpha} \cdot 1_{\{z \neq 0\}} \cdot \bar{z}, \quad \alpha \in(0,1),
$$

and $\tau$ is the coupling time given by

$$
\tau:=\inf \left\{t>0:\left|X_{t}-Y_{t}\right|=0\right\} .
$$

The second equation in (4) can be solved as follows: Define

$$
a_{\varepsilon}(z):=\left|x_{0}-y_{0}\right|^{\alpha} \cdot f_{\varepsilon}(|z|) \cdot \bar{z},
$$

where $f_{\varepsilon}(r): \mathbb{R}_{+} \mapsto[0,1]$ is smooth and satisfies

$$
f_{\varepsilon}(r)=1 \text { for } r>\varepsilon \text {, and } f_{\varepsilon}(r)=0 \text { for } r \in[0, \varepsilon / 2] .
$$

It is easy to see that there is a $C_{\varepsilon}>0$ such that for any $z, z^{\prime} \in \mathbb{R}^{d}$

$$
\left|a_{\varepsilon}(z)-a_{\varepsilon}\left(z^{\prime}\right)\right| \leqslant C_{\varepsilon} \cdot\left|z-z^{\prime}\right| .
$$

So, there is a unique solution $Y_{t}^{\varepsilon}$ to the following SDE:

$$
\mathrm{d} Y_{t}^{\varepsilon}=\left[b\left(Y_{t}^{\varepsilon}\right)+a_{\varepsilon}\left(X_{t}-Y_{t}^{\varepsilon}\right)\right] \mathrm{d} t+\sigma\left(Y_{t}^{\varepsilon}\right) \mathrm{d} W_{t}, \quad Y_{0}^{\varepsilon}=y_{0} .
$$

Define

$$
\tau_{\varepsilon}:=\inf \left\{t>0:\left|X_{t}-Y_{t}^{\varepsilon}\right| \leqslant \varepsilon\right\} .
$$

For $\varepsilon^{\prime}<\varepsilon$, by the uniqueness we clearly have $\tau_{\varepsilon^{\prime}} \geqslant \tau_{\varepsilon}$ and

$$
Y_{t}^{\varepsilon}=Y_{t}^{\varepsilon^{\prime}} \text { on }\left\{t<\tau_{\varepsilon}\right\} \text {. }
$$

Thus, $\tau=\lim _{\varepsilon \downarrow 0} \tau_{\varepsilon}$ is just the coupling time and $Y_{t}$ is well defined on $[0, \tau]$, and we also define

$$
Y_{t}:=X_{t}, \quad \text { for all } t \geqslant \tau .
$$

Then, it is clear that $Y_{t}$ solves the second equation in (4).

Set

$$
Z_{t}:=X_{t}-Y_{t}
$$

Using Itô's formula to the function $r \mapsto \sqrt{|r|^{2}+\varepsilon}$, then letting $\varepsilon \downarrow 0$, we obtain by (H1)

$$
\begin{aligned}
& \left|Z_{t \wedge \tau}\right|-\left|x_{0}-y_{0}\right|-\int_{0}^{t \wedge \tau}\left\langle\bar{Z}_{s},\left(\sigma\left(X_{s}\right)-\sigma\left(Y_{s}\right)\right) \mathrm{d} W_{s}\right\rangle \\
= & \int_{0}^{t \wedge \tau}\left(2\left|Z_{s}\right|\right)^{-1} \cdot\left(2\left\langle Z_{s}, b\left(X_{s}\right)-b\left(Y_{s}\right)\right\rangle+\left\|\sigma\left(X_{s}\right)-\sigma\left(Y_{s}\right)\right\|_{2}^{2}\right) \mathrm{d} s \\
& -\int_{0}^{t \wedge \tau}\left\langle\bar{Z}_{s}, a\left(Z_{s}\right)\right\rangle \mathrm{d} s-\int_{0}^{t \wedge \tau}\left(2\left|Z_{s}\right|\right)^{-1} \cdot\left|\left[\sigma\left(X_{s}\right)-\sigma\left(Y_{s}\right)\right]^{*}\left(\bar{Z}_{s}\right)\right|^{2} \mathrm{~d} s \\
\leqslant & \frac{\lambda_{0}}{2} \int_{0}^{t \wedge \tau}\left|Z_{s}\right|\left(1 \vee \log \left|Z_{s}\right|^{-1}\right) \mathrm{d} s-\left|x_{0}-y_{0}\right|^{\alpha}(t \wedge \tau) .
\end{aligned}
$$

Note that there exists an $\eta>0$ such that

$$
r\left(1 \vee \log r^{-1}\right) \leqslant \rho_{\eta}(r), \quad \forall r>0 .
$$


Taking expectations yields that

$$
\begin{aligned}
\mathbf{E}\left|Z_{t \wedge \tau}\right| & \leqslant\left|x_{0}-y_{0}\right|-\left|x_{0}-y_{0}\right|^{\alpha} \cdot \mathbf{E}(t \wedge \tau)+\frac{\lambda_{0}}{2} \mathbf{E} \int_{0}^{t \wedge \tau} \rho_{\eta}\left(\left|Z_{s}\right|\right) \mathrm{d} s \\
& \leqslant\left|x_{0}-y_{0}\right|-\left|x_{0}-y_{0}\right|^{\alpha} \cdot \mathbf{E}(t \wedge \tau)+\frac{\lambda_{0}}{2} \int_{0}^{t} \rho_{\eta}\left(\mathbf{E}\left|Z_{s \wedge \tau}\right|\right) \mathrm{d} s,
\end{aligned}
$$

where the second step is due to Jensen's inequality.

By the Bihari inequality (3), we get that for any $t>0$ and $\left|x_{0}-y_{0}\right|<\eta$,

$$
\mathbf{E}\left|Z_{t \wedge \tau}\right| \leqslant\left|x_{0}-y_{0}\right|^{\exp \left\{-\lambda_{0} t / 2\right\}}
$$

and

$$
\mathbf{E}(t \wedge \tau) \leqslant\left|x_{0}-y_{0}\right|^{1-\alpha}+\frac{\lambda_{0} t}{2} \rho_{\eta}\left(\left|x_{0}-y_{0}\right|^{\exp \left\{-\lambda_{0} t / 2\right\}}\right) \cdot\left|x_{0}-y_{0}\right|^{-\alpha} .
$$

We now fix a $T>0$ and define

$$
R_{T}:=\exp \left[\int_{0}^{T \wedge \tau}\left\langle\mathrm{d} W_{s}, H\left(X_{s}, Y_{s}\right)\right\rangle-\frac{1}{2} \int_{0}^{T \wedge \tau}\left|H\left(X_{s}, Y_{s}\right)\right|^{2} \mathrm{~d} s\right]
$$

and

where

$$
\tilde{W}_{t}:=W_{t}+\int_{0}^{t \wedge \tau} H\left(X_{s}, Y_{s}\right) \mathrm{d} s
$$

$$
H(x, y):=\left|x_{0}-y_{0}\right|^{\alpha} \cdot[\sigma(y)]^{-1}(\overline{x-y}) .
$$

By (H3), we have

$$
|H(x, y)|^{2} \leqslant \lambda_{2}^{2} \cdot\left|x_{0}-y_{0}\right|^{2 \alpha} .
$$

Thus,

$$
\mathbf{E} R_{T}=1 \quad \text { and } \quad \mathbf{E} R_{T}^{2} \leqslant \exp \left[3 T \lambda_{2}^{2} \cdot\left|x_{0}-y_{0}\right|^{2 \alpha} / 2\right] .
$$

By Girsanov's theorem, $\left(\tilde{W}_{t}\right)_{t \in[0, T]}$ is still a $d$-dimensional Brownian motion under the new probability measure $R_{T} \cdot \mathbf{P}$. Note that $Y_{t}$ also solves

$$
\mathrm{d} Y_{t}=b\left(Y_{t}\right) \mathrm{d} t+\sigma\left(Y_{t}\right) \mathrm{d} \tilde{W}_{t}, \quad Y_{0}=y_{0} .
$$

So, the law of $X_{T}\left(y_{0}\right)$ under $\mathbf{P}$ is the same as the law of $Y_{T}\left(y_{0}\right)$ under $R_{T} \cdot \mathbf{P}$. We thus have for any $\varphi \in B_{b}\left(\mathbb{R}^{d}\right)$

$$
\begin{aligned}
& \left|P_{T} \varphi\left(x_{0}\right)-P_{T} \varphi\left(y_{0}\right)\right| \\
= & \left|\mathbf{E}\left(\varphi\left(X_{T}\left(x_{0}\right)\right)-R_{T} \cdot \varphi\left(Y_{T}\left(y_{0}\right)\right)\right)\right| \\
\leqslant & \mathbf{E}\left|\left(1-R_{T}\right) \cdot \varphi\left(X_{T}\left(x_{0}\right)\right) \cdot 1_{\{\tau \leqslant T\}}\right| \\
& +\mathbf{E}\left|\left(\varphi\left(X_{T}\left(x_{0}\right)\right)-R_{T} \cdot \varphi\left(Y_{T}\left(y_{0}\right)\right)\right) \cdot 1_{\{\tau>T\}}\right| \\
\leqslant & \|\varphi\|_{0} \cdot \mathbf{E}\left|1-R_{T}\right|+\|\varphi\|_{0} \cdot \mathbf{E}\left[\left(1+R_{T}\right) 1_{\{\tau>T\}}\right] .
\end{aligned}
$$

By the elementary inequality $e^{r}-1 \leqslant r e^{r}$ for $r \geqslant 0$, we have for any $\left|x_{0}-y_{0}\right| \leqslant \eta$,

$$
\begin{aligned}
& \left(\mathbf{E}\left|1-R_{T}\right|\right)^{2} \leqslant \mathbf{E}\left|1-R_{T}\right|^{2}=\mathbf{E} R_{T}^{2}-1 \\
\leqslant & \exp \left[3 T \lambda_{2}^{2} \cdot\left|x_{0}-y_{0}\right|^{2 \alpha} / 2\right]-1 \leqslant C_{T, \lambda_{2}, \eta} \cdot\left|x_{0}-y_{0}\right|^{2 \alpha}
\end{aligned}
$$

and

$$
\begin{aligned}
& \left(\mathbf{E}\left[\left(1+R_{T}\right) 1_{\{\tau \geqslant T\}}\right]\right)^{2} \leqslant\left(3+\mathbf{E} R_{T}^{2}\right) \cdot \mathbf{P}(\tau \geqslant T) \\
\leqslant \quad & C_{T, \lambda_{2}, \eta} \cdot \mathbf{P}((2 T) \wedge \tau \geqslant T) \leqslant C_{T, \lambda_{2}, \eta} \cdot \mathbf{E}((2 T) \wedge \tau) / T .
\end{aligned}
$$


Taking $\alpha=\exp \left\{-\lambda_{0} T\right\} / 3$, then by (5) there exists a $0<\eta^{\prime}<\eta$ such that for any $\left|x_{0}-y_{0}\right|<\eta^{\prime}$

$$
\mathbf{E}((2 T) \wedge \tau) \leqslant C_{T, \lambda_{0}, \eta^{\prime}} \cdot\left|x_{0}-y_{0}\right|^{\exp \left\{-\lambda_{0} T\right\} / 2} .
$$

Summarizing the above calculations, we obtain the following strong Feller property:

Theorem 2.2. Under $(\mathbf{H} 1)-(\mathbf{H} 3)$, for any $T>0$, there exist an $\eta>0$ and constant $C_{T, \lambda_{0}, \lambda_{2}, \eta}>0$ such that for all $x_{0}, y_{0} \in \mathbb{R}^{d}$ with $\left|x_{0}-y_{0}\right| \leqslant \eta$ and $\varphi \in B_{b}\left(\mathbb{R}^{d}\right)$,

$$
\left|P_{T} \varphi\left(x_{0}\right)-P_{T} \varphi\left(y_{0}\right)\right| \leqslant C_{T, \lambda_{0}, \lambda_{2}, \eta} \cdot\|\varphi\|_{0} \cdot\left|x_{0}-y_{0}\right|^{\exp \left\{-\lambda_{0} T\right\} / 4} .
$$

2.2. Irreducibility. For proving the irreducibility of $P_{t}$, it suffices to prove that for any $x_{0} \in \mathbb{R}^{d}, T>0$, and $y_{0} \in \mathbb{R}^{d}, a>0$,

$$
P_{T}\left(x_{0}, B\left(y_{0}, a\right)\right)=\mathbf{P}\left(\left|X_{T}\left(x_{0}\right)-y_{0}\right| \leqslant a\right)>0,
$$

where $B\left(y_{0}, a\right):=\left\{z \in \mathbb{R}^{d}:\left|z-y_{0}\right| \leqslant a\right\}$. The $T, x_{0}, y_{0}$ and $a$ will be fixed in what follows.

By $(\mathbf{H} 1)$ and $(\mathbf{H} 2)$, it is a standard deduction that for some $C_{T, x_{0}, \lambda_{0}, \lambda_{1}}>0$

$$
\sup _{t \in[0, T]} \mathbf{E}\left|X_{t}\left(x_{0}\right)\right|^{2} \leqslant C_{T, x_{0}, \lambda_{0}, \lambda_{1}}
$$

Let $t_{1} \in(0, T)$, whose value will be determined below. Set for $\varepsilon>0$

$$
X_{t_{1}}^{\varepsilon}:=X_{t_{1}} \cdot 1_{\left\{\left|X_{t_{1}}\right| \leqslant \varepsilon^{-1}\right\}} .
$$

Then

$$
\lim _{\varepsilon \downarrow 0} \mathbf{E}\left|X_{t_{1}}^{\varepsilon}-X_{t_{1}}\right|^{2}=0
$$

Define for $s \in\left[t_{1}, T\right]$

$$
Y_{s}^{\varepsilon}:=\frac{T-s}{T-t_{1}} X_{t_{1}}^{\varepsilon}+\frac{s-t_{1}}{T-t_{1}} y_{0}
$$

and

$$
h_{s}^{\varepsilon}:=\frac{y_{0}-X_{t_{1}}^{\varepsilon}}{T-t_{1}}-b\left(Y_{s}^{\varepsilon}\right)
$$

Then

$$
Y_{t_{1}}^{\varepsilon}=X_{t_{1}}^{\varepsilon}, \quad Y_{T}^{\varepsilon}=y_{0}
$$

and

$$
Y_{t}^{\varepsilon}=X_{t_{1}}^{\varepsilon}+\int_{t_{1}}^{t} b\left(Y_{s}^{\varepsilon}\right) \mathrm{d} s+\int_{t_{1}}^{t} h_{s}^{\varepsilon} \mathrm{d} s, \quad t \in\left[t_{1}, T\right] .
$$

Consider the following SDE on $\left[t_{1}, T\right]$ :

$$
X_{t}^{\varepsilon}=X_{t_{1}}+\int_{t_{1}}^{t} b\left(X_{s}^{\varepsilon}\right) \mathrm{d} s+\int_{t_{1}}^{t} h_{s}^{\varepsilon} \mathrm{d} s+\int_{t_{1}}^{t} \sigma\left(X_{s}^{\varepsilon}\right) \mathrm{d} W_{s} .
$$

If we define

$$
X_{t}^{\varepsilon}:=X_{t}, \quad \forall t \in\left[0, t_{1}\right],
$$

then for any $t \in[0, T]$

$$
X_{t}^{\varepsilon}=x_{0}+\int_{0}^{t} b\left(X_{s}^{\varepsilon}\right) \mathrm{d} s+\int_{0}^{t} 1_{\left\{s>t_{1}\right\}} h_{s}^{\varepsilon} \mathrm{d} s+\int_{0}^{t} \sigma\left(X_{s}^{\varepsilon}\right) \mathrm{d} W_{s} .
$$


We now define

$$
\tilde{W}_{t}^{\varepsilon}:=W_{t}+\int_{0}^{t} H_{s}^{\varepsilon} \mathrm{d} s
$$

and

$$
R_{T}^{\varepsilon}:=\exp \left[\int_{0}^{T}\left\langle\mathrm{~d} W_{s}, H_{s}^{\varepsilon}\right\rangle-\frac{1}{2} \int_{0}^{T}\left|H_{s}^{\varepsilon}\right|^{2} \mathrm{~d} s\right],
$$

where

$$
H_{s}^{\varepsilon}:=1_{\left\{s>t_{1}\right\}}\left[\sigma\left(X_{s}^{\varepsilon}\right)\right]^{-1} h_{s}^{\varepsilon} .
$$

Noting that by (H3), (8), (10) and the continuity of $b$

$$
\left|H_{s}^{\varepsilon}\right| \leqslant \lambda_{2}\left|h_{s}^{\varepsilon}\right| \leqslant C_{\lambda_{2}, \varepsilon, t_{1}}
$$

we have

$$
\mathbf{E} R_{T}^{\varepsilon}=1, \quad \mathbf{P}\left(R_{T}^{\varepsilon}>0\right)=1,
$$

and $\left(\tilde{W}_{t}^{\varepsilon}\right)_{t \in[0, T]}$ is a $d$-dimensional Brownian motion under the new probability measure $R_{T}^{\varepsilon} \cdot \mathbf{P}$. Thus, $X_{T}^{\varepsilon}\left(x_{0}\right)$ has the same law as $X_{T}\left(x_{0}\right)$. Hence, in order to prove (6), it suffices to prove that for some $t_{1} \in(0, T)$ and $\varepsilon>0$

$$
\mathbf{P}\left(\left|X_{T}^{\varepsilon}\left(x_{0}\right)-y_{0}\right| \leqslant a\right)>0,
$$

or equivalently,

$$
\mathbf{P}\left(\left|X_{T}^{\varepsilon}\left(x_{0}\right)-y_{0}\right|>a\right)<1 .
$$

Set

$$
Z_{t}^{\varepsilon}:=X_{t}^{\varepsilon}-Y_{t}^{\varepsilon}
$$

By Itô's formula, we have by (H1) and (H2)

$$
\begin{aligned}
\mathbf{E}\left|Z_{t}^{\varepsilon}\right|^{2}= & \mathbf{E}\left|X_{t_{1}}-X_{t_{1}}^{\varepsilon}\right|^{2}+\int_{t_{1}}^{t} \mathbf{E}\left(2\left\langle Z_{s}^{\varepsilon}, b\left(X_{s}^{\varepsilon}\right)-b\left(Y_{s}^{\varepsilon}\right)\right\rangle+\left\|\sigma\left(X_{s}^{\varepsilon}\right)\right\|_{2}^{2}\right) \mathrm{d} s \\
\leqslant & \mathbf{E}\left|X_{t_{1}}-X_{t_{1}}^{\varepsilon}\right|^{2}+C_{\kappa_{0}} \int_{t_{1}}^{t} \mathbf{E}\left(\left|Y_{s}^{\varepsilon}\right|^{2}+1\right) \mathrm{d} s \\
& +C_{\lambda_{0}, \kappa_{0}} \int_{t_{1}}^{t} \mathbf{E}\left(\left|Z_{s}^{\varepsilon}\right|^{2}\left(1 \vee \log \left|Z_{s}^{\varepsilon}\right|^{-1}\right)\right) \mathrm{d} s
\end{aligned}
$$

Noticing that by (8) and (7)

$$
\begin{aligned}
\int_{t_{1}}^{T} \mathbf{E}\left|Y_{s}^{\varepsilon}\right|^{2} \mathrm{~d} s & \leqslant 2\left(T-t_{1}\right) \cdot\left(\mathbf{E}\left|X_{t_{1}}^{\varepsilon}\right|^{2}+\left|y_{0}\right|^{2}\right) \\
& \leqslant 2\left(T-t_{1}\right) \cdot\left(C_{T, x_{0}, \lambda_{0}, \lambda_{1}}+\left|y_{0}\right|^{2}\right),
\end{aligned}
$$

and there exists an $\eta>0$ such that

$$
r^{2}\left(1 \vee \log r^{-1}\right) \leqslant \rho_{\eta}\left(r^{2}\right), \quad \forall r>0,
$$

we have by Jensen's inequality

$$
\mathbf{E}\left|Z_{t}^{\varepsilon}\right|^{2} \leqslant \mathbf{E}\left|X_{t_{1}}-X_{t_{1}}^{\varepsilon}\right|^{2}+C\left|T-t_{1}\right|+C_{\lambda_{0}, \kappa_{0}} \int_{t_{1}}^{t} \rho_{\eta}\left(\mathbf{E}\left|Z_{s}^{\varepsilon}\right|^{2}\right) \mathrm{d} s,
$$

where $C$ is independent of $\varepsilon$ and $t_{1}$.

By the Bihari inequality (3) again, we obtain

$$
\mathbf{E}\left|X_{T}^{\varepsilon}-y_{0}\right|^{2} \leqslant\left[\mathbf{E}\left|X_{t_{1}}-X_{t_{1}}^{\varepsilon}\right|^{2}+C\left(T-t_{1}\right)\right]^{\exp \left\{-C_{\lambda_{0}, \kappa_{0}} \cdot T\right\}} .
$$


Hence,

$$
\begin{aligned}
& \mathbf{P}\left(\left|X_{T}^{\varepsilon}\left(x_{0}\right)-y_{0}\right|>a\right) \leqslant \frac{1}{a^{2}} \mathbf{E}\left|X_{T}^{\varepsilon}\left(x_{0}\right)-y_{0}\right|^{2} \\
\leqslant & \frac{1}{a^{2}} \cdot\left[\mathbf{E}\left|X_{t_{1}}-X_{t_{1}}^{\varepsilon}\right|^{2}+C\left(T-t_{1}\right)\right]^{\exp \left\{-C_{\lambda_{0}, \kappa_{0}} \cdot T\right\}} .
\end{aligned}
$$

First letting $t_{1}$ close to $T$, and then choosing $\varepsilon$ to be sufficiently small, we obtain by (9)

$$
\mathbf{P}\left(\left|X_{T}^{\varepsilon}\left(x_{0}\right)-y_{0}\right|>a\right)<1 .
$$

The proof of irreducibility is thus complete.

2.3. Proof of Theorem 1.1. We now assume that $(\mathbf{H} 1)-(\mathbf{H} 4)$ hold. Using Itô's formula, we have by (H4) and Hölder's inequality

$$
\begin{aligned}
\frac{\mathrm{d} \mathbf{E}\left|X_{t}\right|^{2}}{\mathrm{~d} t} & =\mathbf{E}\left(2\left\langle X_{t}, b\left(X_{t}\right)\right\rangle+\left\|\sigma\left(X_{t}\right)\right\|_{2}^{2}\right) \\
& \leqslant-\lambda_{3} \mathbf{E}\left|X_{t}\right|^{p}+\lambda_{4} \\
& \leqslant-\lambda_{3}\left(\mathbf{E}\left|X_{t}\right|^{2}\right)^{p / 2}+\lambda_{4} .
\end{aligned}
$$

Hence, for all $t>0$

$$
\frac{1}{t} \int_{0}^{t} \mathbf{E}\left|X_{s}\right|^{2} \mathrm{~d} s \leqslant \lambda_{4}
$$

The existence of an invariant probability measure $\mu$ now follows from the classical Krylov-Bogoliubov's method (cf. 2]). The first conclusion follows from the strong Feller property and irreducibility (cf. 9, 14, 2]).

On the other hand, if $\lambda_{3}>0$, let $f(t)$ solve the following ODE:

$$
f^{\prime}(t)=\lambda_{4}-\lambda_{3} f(t)^{p / 2}, \quad f(0)=\left|x_{0}\right|^{2} .
$$

By the comparison theorem in ODE and [2, Lemma 1.2.6], we have for some $C>0$

$$
\mathbf{E}\left|X_{t}\right|^{2} \leqslant f(t) \leqslant C\left[1+t^{\frac{1}{1-p / 2}}\right],
$$

where the right hand side is independent of $x_{0}$.

Since $P_{t}$ is strong Feller and irreducible, we also have for any $r, a>0$ and $t>0$

$$
\inf _{x_{0} \in B(0, r)} P_{t}\left(x_{0}, B(0, a)\right)>0 .
$$

The second conclusion then follows from [6, Theorem 2.5 (b) and Theorem 2.7].

\section{REFERENCES}

[1] Airault, H. and Ren, J.: Modulus of continuity of the canonic Brownian motion "on the group of diffeomorphisms of the circle", J. Funct. Anal., 196/2 (2002), 395-426. MR1943096 (2003i:58066)

[2] Cerrai, S.: Second order PDE's in finite and infinite dimension. A probabilistic approach, Lecture Notes in Mathematics, 1762. Springer-Verlag, Berlin, 2001. x+330 pp. MR1840644 (2002j:35327)

[3] Da Prato, G. and Zabczyk, J.: Ergodicity for Infinite Dimensional Systems. Cambridge University Press, 1996. MR.1417491 (97k:60165)

[4] Elworthy, K.D. and Li, X.M.: Formulae for the derivatives of heat semigroup, J. Func. Anal., 125(1) (1994), 252-286. MR.1297021 (95j:60087)

[5] Fang, S., Zhang, T.: Isotropic stochastic flow of homeomorphisms on $S^{d}$ for the critical Sobolev exponent, J. Math. Pures Appl. (9) 85 (2006), no. 4, 580-597. MR2216308 (2007i:60067) 
[6] Goldys, B. and Maslowski, B.: Exponential ergodicity for stochastic reaction-diffusion equations, Stochastic partial differential equations and applications-VII, 115-131, Lect. Notes Pure Appl. Math., 245, Chapman Hall/CRC, Boca Raton, FL, 2006. MR2227225 (2007g:60088)

[7] LeJan, Y. and Raimond, O.: Integration of Brownian vector fields. Ann. of Prob., 30 (2002), 826-873. MR1905858(2003d:60114)

[8] Malliavin, P.: The canonic diffusion above the diffeomorphism group of the circle, C.R. Acad. Sci. Paris, Série I, 329 (1999), pp. 325-329. MR1713340(2000e:60129)

[9] Meyn, S. P. and Tweedie, R. L.: Markov chains and stochastic stability, Communications and Control Engineering Series. Springer-Verlag London, Ltd., London, 1993. MR 1287609 (95j:60103)

[10] Ren, J. and Zhang, X.: Freidlin-Wentzell's large deviations for homeomorphism flows of non-Lipschitz SDEs, Bull. Sci. Math. 129/8 (2005), 643-655. MR2166732 (2006h:60102)

[11] Ren, J. and Zhang, X.: Continuity Modulus of stochastic homeomorphism flows for SDEs with non-Lipschitz coefficients, J. Func. Anal., 241, (2) (2006), 439-456. MR2271926

[12] Ren, J. and Zhang, X.: Large deviations of stochastic flows for non-Lipschitz SDEs in modulus spaces, preprint.

[13] Wang, F.Y.: Harnack Inequality and Applications for Stochastic Generalized Porous Media Equations, to appear in Annals. of Probability.

[14] Stettner, L.: Remarks on ergodic conditions for Markov processes on Polish spaces, Bull. Polish Acad. Sci. Math., 42 (2) (1994), 103-114. MR1810695

[15] Zhang, X.: Homeomorphic flows for multi dimensional SDEs with non-Lipschitz coefficients, Stochastic Processes and their Applications, 115 (2005), 435-448; 116 (2006), 873-875. MR2118287 (2006b:60132), MR2218340 (2006k:60108)

Department of Mathematics, Huazhong University of Science and Technology, Wuhan, Hubei 430074, People's Republic of China

Current address: School of Mathematics and Statistics, The University of New South Wales, Sydney, NSW 2052, Australia

E-mail address: XichengZhang@gmail.com 